\title{
Impact of the use of vasoactive drugs in cardiac death donors on the early postoperative renal function and related complications in renal transplant recipients
}

\author{
Peng Zhang ${ }^{1}$, Peihua Cao ${ }^{2}$, Jiali Fang ${ }^{1}$, Guanghui Li $^{1}$, Lei Zhang ${ }^{1}$, Lu Xu ${ }^{1}$, Shijing Mo ${ }^{3}$, Xingqiang Lai ${ }^{1}$, \\ Luhao Liu ${ }^{1}$, Yunyi Xiong ${ }^{1}$, Wei Yin ${ }^{1}$, Li Li ${ }^{1}$, Rongxin Chen ${ }^{1}$, Hailin Xu', Tao Zhang ${ }^{1}$, Jiao Wan ${ }^{1}$, Yuhe Guo ${ }^{1}$, \\ Junjie $\mathrm{Ma}^{1}$, Zheng Chen ${ }^{1}$ \\ ${ }^{1}$ Organ Transplant Center, the Second Affiliated Hospital of Guangzhou Medical University, Guangzhou511447, China; ${ }^{2}$ Clinical Research Center, \\ Zhujiang Hospital, Southern Medical University, Guangzhou 510280, China; ${ }^{3}$ Department of Intensive Care Unit, Zhejiang Provincial People's \\ Hospital, People's Hospital of Hangzhou Medical College, Hangzhou 310014, China \\ Contributions: (I) Conception and design: P Zhang;(II) Administrative support: Z Chen, J Ma; (III) Provision of study materials or patients: G Li, J \\ Fang, L Zhang, L Xu; (IV) Collection and assembly of data: X Lai, S Mo, L Liu, Y Xiong, L Li, W Yin, R Chen, J Wan, Y Guo, H Xu, T Zhang; (V) \\ Data analysis and interpretation: P Zhang, P Cao, Z Chen; (VI) Manuscript writing: All authors; (VII) Final approval of manuscript: All authors. \\ Correspondence to: Zheng Chen, MD. Professor, Organ Transplant Center, the Second Affiliated Hospital of Guangzhou Medical University, \\ Guangzhou 511447, China. Email: docchenzheng@163.com.
}

Background: To explore the impact of the use of vasoactive drugs in donation after cardiac death (DCD) donors on graft function, with an attempt to guide the clinical practices of organ preservation and DCD kidney transplantation.

Methods: The clinical data of 187 DCD donors and 304 recipients who were operated on in our center from February 2018 to May 2019 were retrospectively analyzed. Based on whether vasoactive drugs were used for maintaining blood pressure in DCD donors, the renal donors and recipients were divided into a high-dose group (norepinephrine $\geq 1.3 \mu \mathrm{g} / \mathrm{kg} / \mathrm{min}$ or in combination with dopamine), a low-dose group (norepinephrine $<1.3 \mu \mathrm{g} / \mathrm{kg} / \mathrm{min}$ or in conjunction with dopamine), and a no-medication group (without the use of vasoactive drugs). The clinical features, post-transplant renal function, and complications were compared among these three groups.

Results: The early renal function 1 and 7 days after surgery was significantly superior in the high-dose group and no-medication group $(\mathrm{P}<0.05)$ but showed no significant difference between the low-dose group and the no-medication group $(\mathrm{P}>0.05)$. Blood urea nitrogen $(\mathrm{BUN})$ on the 1 st postoperative days was significantly higher in the high-dose group than in the low-dose group and the no-medication group $(\mathrm{P}<0.05)$. Renal function indicators, including serum creatinine (CRE), BUN, and blood uric acid (UA) on the 30th postoperative day, showed no significant difference among these three groups $(\mathrm{P}>0.05)$. The incidence of delayed graft function (DGF) after renal transplantation was significantly higher in the high-dose group than in the low-dose group and the no-medication group $(\mathrm{P}<0.05)$, whereas there was no significant difference between the groups in the incidences of graft rejection and infections $(\mathrm{P}>0.05)$.

Conclusions: The use of vasoactive drugs in DCD donors can affect the early recovery of renal function in renal transplant recipients, particularly for those donors who are administered a high dose of vasoactive drugs. Therefore, donor maintenance should be performed cautiously with vasoactive drugs.

Keywords: Kidney transplantation; donation after cardiac death (DCD); vasoactive drugs; complications

Submitted Oct 14, 2019. Accepted for publication Nov 12, 2019.

doi: 10.21037/atm.2019.12.99

View this article at: http://dx.doi.org/10.21037/atm.2019.12.99 


\section{Introduction}

Donation after cardiac death (DCD), an essential source of organs for alleviating organ shortages, has been recognized by the World Health Organization and adopted worldwide. However, the hemodynamic instability after donor brain death can lead to donation failure in $25 \%$ of potential donors (1). Therefore, the use of vasoactive drugs to support blood pressure is required in hypotensive donors whose hemodynamic status remains unstable even after adequate rehydration therapy. It has been reported that more than $80 \%$ of potential donors require vasoactive drugs to support their hemodynamic stability (2). However, the role of vasoactive drugs in organ preservation is still controversial. Based on the organ donation and preservation in our center, we retrospectively analyzed the clinical data of 187 DCD renal donors in our center from February 2018 to May 2015. Our aim was to explore the impact of the use of vasoactive drugs in postoperative renal function and related complications in renal transplant recipients, and thus to further inform the practices of donor maintenance and DCD renal transplantation.

\section{Methods}

\section{Subjects}

\section{General data}

The clinical data of 187 DCD donors who were operated on in our center from February 2018 to May 2019 were retrospectively analyzed. According to the use of vasoactive drugs, these DCD donors were divided into a high-dose group, a low-dose group, and a no-medication group.

\section{Grouping}

Based on whether vasoactive drugs were used or not in DCD donors before organ procurement, the renal donor $(n=187)$ and recipients $(\mathrm{n}=304)$ were divided into a high-dose group (norepinephrine $\geq 1.3 \mu \mathrm{g} / \mathrm{kg} / \mathrm{min}$ or in combination with dopamine), a low-dose group (norepinephrine $<1.3 \mu \mathrm{g} / \mathrm{kg} / \mathrm{min}$ or in combination with dopamine), and a no-medication group (without the use of vasoactive drugs). The general data of the donors and recipients, along with the renal function and complications after kidney transplantation, were observed and compared among these three groups.

\section{Kidney procurement and transplantation}

The acquisition of DCD kidneys was conducted under the supervision of the Human Organ Donation Office. The donors met the diagnostic criteria for cardiac death in the Chinese Guidelines on the Donation of Cardiac Death Organs (3), and organ procurement began 2 minutes after cardiac death. After a large abdominal median incision was made, the superior mesenteric vein, abdominal aorta, and inferior vena cava were cannulated. When the abdominal aorta and superior mesenteric vein were perfused with $3000 \mathrm{ml}$ of hypertonic citrate flushing solution for kidney preservation, the University of Wisconsin (UW) solution was applied (1 bag in each vessel). After the tissues were mobilized, the distal ureter was disconnected, and the kidneys were harvested. Kidney transplant recipient surgery was performed in routine fashion. After the DCD kidney was transplanted into the axillary fossa of the recipient, the artery of the donor's kidney was anastomosed with the external iliac artery of the recipient (or end-to-end anastomosis with the internal iliac artery), the vein of the donor's kidney was anastomosed to the external iliac vein in an end-to-side manner, and the ureter of the donor was submucosally tunneled with the recipient's bladder, and accompanied by routine double J (DJ) ureteral stenting. After surgery, all patients were administered with triple immunotherapy, including tacrolimus (or cyclosporine), mycophenolate mofetil, and glucocorticoid.

\section{Main measures}

\section{DCD donors}

Information collected from donors included age, gender, body mass index (BMI), mean arterial pressure, primary disease, duration of ICU stay, pre-donation creatinine level, and use of vasoactive drugs.

\section{Intraoperative and postoperative observation indicators} of recipients

Meanwhile, the intraoperative and postoperative observation indicators of recipients included age, gender, BMI, times of transplants, dialysis method, duration of dialysis, intraoperative blood loss, postoperative renal function recovery, and complications.

\section{Renal function indicators of recipients after kidney transplantation}

Changes in serum creatinine (CRE), blood urea nitrogen (BUN), and blood uric acid (UA) before and 1, 7, and 30 days after renal transplantation were compared. Postoperative complications, including delayed graft 
Table 1 Data of donors

\begin{tabular}{|c|c|c|c|c|}
\hline Age & $42.0 \pm 10.6$ & $41.0 \pm 13.7$ & $37.8 \pm 15.6$ & 0.352 \\
\hline Gender & & & & 0.175 \\
\hline Male & 17 & 110 & 41 & \\
\hline BMI & $23.3 \pm 2.7$ & $23.2 \pm 3.7$ & $22.4 \pm 3.7$ & 0.387 \\
\hline Primary disease & & & & 0.33 \\
\hline $\begin{array}{l}\text { Cerebrovascular diseases (cerebral hemorrhage or } \\
\text { cerebral infarction) }\end{array}$ & 7 & 58 & 26 & \\
\hline Others & 1 & 4 & 3 & \\
\hline Length of ICU stay (day) & $5.3 \pm 4.0$ & $8.1 \pm 12.0$ & $7.4 \pm 7.4$ & 0.523 \\
\hline Serum creatinine $(\mu \mathrm{mol} / \mathrm{L})$ & $160.9 \pm 91.4$ & $144.0 \pm 92.1$ & $139.3 \pm 82.4$ & 0.67 \\
\hline
\end{tabular}

BMI, body mass index; SD, standard deviations.

function (DGF), graft rejection, and lung or urinary tract infection in the recipients, were recorded. The recovery was regarded as good if serum CRE decreased by over $200 \mu \mathrm{mol} / \mathrm{L}$. DGF was defined as the recipient having oliguria and/or anuria 1 week after transplantation, a serum CRE level $>400 \mu \mathrm{mol} / \mathrm{L}$, and/or requiring hemodialysis.

\section{Statistical analysis}

Statistical analysis was completed with the SPSS 21.0 software package. The count data are presented as the rate $(\%)$ and were analyzed by using the chi-square test. The normal distributed measurement data are expressed as mean \pm standard deviations $(\mathrm{X} \pm \mathrm{SD})$ and analyzed by using the analysis of variance; the non-normally distributed measurement data are expressed as medians (quartiles) and were analyzed by using nonparametric methods. The changes of relevant indicators in the recipients after renal transplantation were analyzed by covariance analysis, with the preoperative findings as covariates. Univariate and multivariate logistic regression analyses were used to investigate the influencing factors of CRE recovery 1 day after renal transplantation. A $\mathrm{P}$ value of $<0.05$ was considered statistically significant.

\section{Results}

\section{General data of the donors}

Among the 187 DCD donors, there were 168 males and 19 females aged 2-64 years (mean: 13.9 \pm 23.0 years), with a BMI value of $23.0 \pm 3.6$. The primary disease among these donors included cerebral hemorrhage $(\mathrm{n}=91)$, severe craniocerebral trauma $(\mathrm{n}=88)$, and other etiologies, including brain tumor, Japanese encephalitis, and organophosphorus pesticide poisoning $(n=8)$. Most donors were kept in the ICU of our hospital. The length of ICU stays ranged from 1-120 days (mean: 7.6 10.4 days). The pre-procurement CRE level was $144.4 \pm 89.4$. Notably, 166 donors required vasoactive drugs to support their hemodynamic stability. Generally, vasoactive drugs include norepinephrine, adrenaline, dopamine, and dobutamine, and in our center, the vasoactive drugs used were norepinephrine and/or dopamine. Among these 187 donors, 47 were in the high-dose group, 119 in the low-dose group, and 21 in the no-medication group. All the donors were comparable in terms of age, gender, and length of ICU stay $(\mathrm{P}>0.05)$ (Table 1).

\section{General data of the recipients}

In total, 304 patients underwent renal transplantation 
Table 2 Data of recipients

\begin{tabular}{|c|c|c|c|c|}
\hline Variable & $\begin{array}{l}\text { No-medication group } \\
\qquad(\mathrm{n}=37)\end{array}$ & $\begin{array}{l}\text { Small-dose group } \\
\qquad(\mathrm{n}=191)\end{array}$ & $\begin{array}{l}\text { High-dose group } \\
\qquad(n=76)\end{array}$ & $P$ value \\
\hline Age (years) & $41.6 \pm 14.5$ & $44.6 \pm 12.7$ & $44.5 \pm 13.2$ & 0.451 \\
\hline Gender & & & & 0.464 \\
\hline Male & 27 & 124 & 52 & \\
\hline BMI & $23.5 \pm 3.8$ & $22.5 \pm 3.6$ & $22.1 \pm 3.3$ & 0.135 \\
\hline Dialysis method & & & & 0.612 \\
\hline None & 4 & 37 & 10 & \\
\hline \multicolumn{5}{|l|}{ Yes } \\
\hline Intraoperative blood loss (mL) & $60.6 \pm 32.2$ & $62.2 \pm 41.5$ & $66.9 \pm 104.0$ & 0.843 \\
\hline
\end{tabular}

BMI, body mass index.

during the study period. These patients included 206 men and 104 women aged $44.2 \pm 13.0$ years, with a mean BMI value of $22.5 \pm 3.6$. Before the operation, 193 had undergone hemodialysis, and 61 had received peritoneal dialysis. The average dialysis time was $21.2 \pm 27.0$ months. According to the use of vasoactive drugs in the donor, the recipients were also divided into the high-dose group, low-dose group, and no-medication group. Among these 304 recipients, 76 were in the high-dose group, 191 in the low-dose group, and 37 in the no-medication group. All the recipients were comparable in terms of age, gender, BMI, method, and duration of dialysis, and intra-operative blood loss $(\mathrm{P}>0.05)$ (Table 2).

\section{Impact of the use of vasoactive drugs in donors on graft function}

CRE, BUN, and serum UA were used to evaluate preoperative and postoperative renal function (Table 3). The preoperative CRE, BUN, and UA levels showed no significant differences among these three groups. Covariance analysis with the preoperative findings as covariates showed that the CRE on the 1 st and 7 th postoperative days was significantly higher in the high-dose group than in the low-dose group and the no-medication group $(\mathrm{P}<0.05)$, whereas there was no significant difference between the low-dose group and the no-medication group ( $\mathrm{P}>0.05)$; BUN was also significantly higher in the high-dose group than in the low-dose group and the nomedication group $(\mathrm{P}<0.05)$. These three groups showed no significant difference in renal function 30 days after surgery $(\mathrm{P}>0.05)$.

\section{Impact of the use of vasoactive drugs on postoperative complications}

Complications after renal transplantation were medical complications, including DGF, graft rejection, and infections. Two patients died of severe pneumonia despite active treatment, and the complications were clinically cured in the remaining patients. The incidence of DGF in the recipients was $22.4 \%$ (17 of 76 ) in high-dose group, which was significantly higher than that in the low-dose group and no-medication group $(\mathrm{P}<0.05)$; the incidences of acute graft rejection and infections were not significantly different among the three groups $(\mathrm{P}>0.05)$ (Table 4).

\section{Influencing factors of CRE recovery 1 day after renal transplantation}

As shown in Table 3, the changes in CRE were most apparent on the first post-operative day in the three groups. Therefore, univariate and multivariate logistic regression analyses with CRE recovery 1 day after renal transplantation 
Table 3 Kidney function recovery early after surgery in renal transplantation recipients $\left(X_{ \pm} S D\right)$

\begin{tabular}{|c|c|c|c|c|}
\hline Variable & No-medication group $(n=37)$ & Small-dose group $(n=119)$ & High-dose group $(n=76)$ & $P$ value \\
\hline Before surgery & $1,026.9 \pm 461.9$ & $975.7 \pm 365.8$ & $1,034.3 \pm 461.0$ & 0.557 \\
\hline The 1st post-op day & $723.3 \pm 299.9^{\#}$ & $749.3 \pm 346.5^{\#}$ & $922.3 \pm 295.6$ & $<0.001$ \\
\hline The 7th post-op day & $224.8 \pm 207.5$ & $225.8 \pm 240.9^{\#}$ & $312.8 \pm 264.3$ & 0.03 \\
\hline \multicolumn{5}{|l|}{ BUN } \\
\hline Before surgery & $23.3 \pm 8.3$ & $24.4 \pm 39.6$ & $28.7 \pm 48.1$ & 0.729 \\
\hline The 1st post-op day & $23.3 \pm 7.2$ & $21.5 \pm 7.6^{\#}$ & $23.9 \pm 6.4$ & 0.041 \\
\hline The 7th post-op day & $17.6 \pm 13.2$ & $18.2 \pm 11.9$ & $20.1 \pm 12.2$ & 0.475 \\
\hline Before surgery & $440.2 \pm 86.4$ & $444.5 \pm 122.0$ & $425.8 \pm 104.2$ & 0.856 \\
\hline The 1st post-op day & $492.5 \pm 125.5$ & $448.1 \pm 153.2$ & $457.2 \pm 132.4$ & 0.487 \\
\hline The 7th post-op day & $351.1 \pm 99.8$ & $388.7 \pm 143.6$ & $374.1 \pm 136.0$ & 0.397 \\
\hline The 30th post-op day & $370.5 \pm 73.0$ & $364.5 \pm 97.2$ & $345.6 \pm 87.2$ & 0.373 \\
\hline
\end{tabular}

\#, Pairwise comparison; $P<0.05$, vs. high-dose group. CRE, serum creatinine; BUN, blood urea nitrogen; UA, serum uric acid; SD, standard deviations.

Table 4 Post-operative complications in recipients among the three groups

\begin{tabular}{lcccc}
\hline Variable & $\begin{array}{c}\text { No-medication group } \\
(\mathrm{n}=37)\end{array}$ & Low-dose group $(\mathrm{n}=191)$ & High-dose group $(\mathrm{n}=76)$ & $\chi^{2}$ \\
\hline Delayed graft function & $3(8.1 \%)$ & $21(11.0 \%)$ & $17(22.4 \%)$ & 6.387 \\
Acute graft rejection & $13(35.1 \%)$ & $57(29.8 \%)$ & $18(23.7 \%)$ & 0.037 \\
Pneumonia or urinary & $1(2.7 \%)$ & $4(2.1 \%)$ & $3(3.9 \%)$ & 0.408 \\
tract infection & & & & 0.669 \\
\hline
\end{tabular}

as the dichotomous variable were performed to investigate the impact of the use of vasoactive drugs of the donors on CRE recovery 1 day after renal transplantation; meanwhile, the recipient characteristics, donor characteristics, and other factors were adjusted. Univariate analysis revealed that, compared with the no-medication group, the lowdose group had significantly improved CRE recovery 1 day after renal transplantation in recipients $(\mathrm{OR}=4.244,95 \%$ CI: 1.803-9.994, $\mathrm{P}=0.001$ ), while there was no significant difference between the high-dose group and the nomedication group (OR $=2.083,95 \%$ CI: $0.815-5.323$, $\mathrm{P}=0.125)$. In addition, the donor's CRE level and length of ICU stay also affected the CRE recovery 1 day after renal transplantation. Multivariate analysis showed that, after the donor's age, CRE level, length of ICU stay, and other factors were adjusted, the CRE recovery 1 day after renal transplantation was significantly improved in the lowdose group (compared with the no-medication group) (OR $=2.998,95 \%$ CI: $1.166-7.709, \mathrm{P}=0.023$ ) (Table 5).

\section{Discussion}

Kidney transplantation has become the ideal alternative treatment for patients with end-stage renal disease, and the 1 -year graft survival rate in large-scale transplant centers in China has exceeded 95\% (4). However, the long-term 
Table 5 Influencing factors of serum creatinine recovery 1 day after renal transplantation (logistic regression analysis)

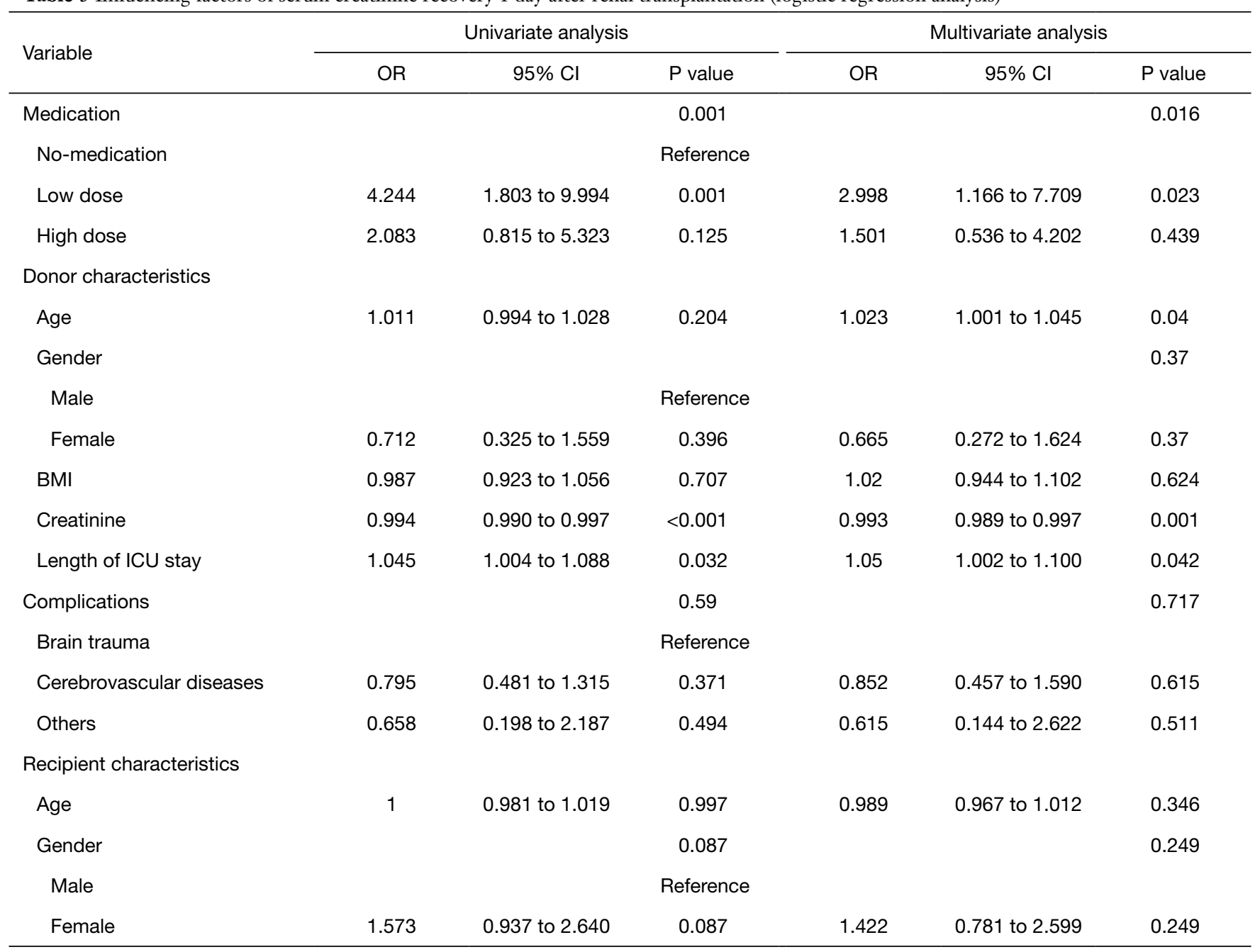

survival rate of renal transplant recipients is still suboptimal. Since the launch of the DCD by the Chinese Red Cross Society and the former Ministry of Health of China on March 2010, the donation and transplantation of DCD organs have developed rapidly in China. Today, DCD donors have become an essential source of organs in China.

However, the dysfunction of sympathetic and parasympathetic nerves after brain death in DCD donors leads to dysregulation of vascular tone, unstable hemodynamics, and decreased blood pressure, causing the warm ischemia of the organ. As extended hypotension will increase the risk of primary non-function, the hemodynamic instability after donor brain death can lead to donation failure in $25 \%$ of the potential donors, and more than $80 \%$ of potential donors require vasoactive drugs to support their hemodynamic stability $(5,6)$. Therefore, enhanced donor maintenance and the optimized use of vasoactive drugs are vital for graft function recovery and long-term graft survival.

Despite this critical need, the role of vasoactive drugs in organ preservation is still controversial (7-9). Researchers believe that vasoactive drugs can support circulation stability and thus protect the grafts. Schnuelle et al. (10) proved that dopamine pretreatment reduced the risk of hemodialysis after renal transplantation, although the long-term survival rate of the grafts was not significantly different between the dopamine and dopamine-free groups $(\mathrm{P}>0.05)$. A study of 152 kidney transplant recipients proved that the use of catecholamines in donors reduced graft rejection and increased the long-term survival of transplanted kidneys (11). Birtan et al. (12), in their Pearson correlation analysis, found that the use of norepinephrine in donor maintenance 
was associated with decreased graft rejection and a lower incidence of graft dysfunction. On the contrary, other researchers have argued that vasoactive drugs harm graft function. O'Brien et al. (13) found that vasoactive drugs increased the incidence of acute tubular necrosis after renal transplantation. In 2012, Shao et al. (14) evaluated the risk factors of renal transplantation and concluded that $72.2 \%$ of the donors in the DGF group had used norepinephrine, whereas only $10 \%$ of donors in the rapid recovery graft function group had used norepinephrine.

Given this lack of consensus, we retrospectively analyzed 187 DCD donors and 304 renal transplant recipients in our center between February 2018 and May 2019. Our goal was to clarify the impact of vasoactive drugs in DCD donors on the early postoperative renal function and related complications in renal transplant recipients. It was found that the CRE on the 1st and 7th postoperative days was significantly higher in the high-dose group than in the low-dose group and the no-medication group $(\mathrm{P}<0.05)$. The BUN was also significantly higher in the high-dose group than in the low-dose group and the no-medication group $(\mathrm{P}<0.05)$, whereas there was no significant difference between the low-dose group and the no-medication group $(\mathrm{P}>0.05)$. These three groups showed no significant difference in renal function 30 days after surgery $(\mathrm{P}>0.05)$ (Table 3). In addition, $22.4 \%$ (17/76) of patients in the high-dose group developed DGF, which was a significantly higher rate than that in the low-dose group $(11.0 \%)$ and the no-medication group $(8.1 \%)(\mathrm{P}<0.05)$. There were no significant differences among these three groups in terms of acute rejection and infections $(\mathrm{P}>0.05)$ (Table 4$)$.

In our current study, we also divided the renal transplant recipients into the good recovery group (CRE decreased by more than $200 \mu \mathrm{mol} / \mathrm{L}$ ) and the poor recovery group (CRE decreased by less than $200 \mu \mathrm{mol} / \mathrm{L}$ ) according to the change of CRE 1 day after kidney transplantation, and analyzed its influencing factors. Preliminary data showed that the doses of vasoactive drugs, donor creatinine levels, and donor ICU stay significantly differed between these two groups $(\mathrm{P}<0.05)$. Multivariate logistic regression analysis showed that the use of low-dose vasoactive drugs and extended ICU stay of the donors were independent protective factors for CRE recovery 1 day after renal transplantation $(\mathrm{P}<0.05)$, whereas high donor creatinine level was an independent risk factor $(\mathrm{P}<0.05)$.

In summary, the use of vasoactive drugs in DCD donors can affect the early recovery of renal function in renal transplant recipients. However, the use of vasoactive high- dose medicine has no remarkable impact on long-term graft function recovery. Thus, the timing of using vasoactive drugs in response to donor blood pressure fluctuation, along with the duration of vasoactive drug use (which may affect the quality of the grafts) warrants further investigation.

\section{Acknowledgments}

Funding: This study was supported by the Guangdong Provincial Natural Science Foundation (2018A030313434), the Guangzhou Municipal Science and Technology Program (805275264049), and the Guangdong Provincial Medical Science Research Foundation (A2018259).

\section{Footnote}

Conflicts of Interest: The authors have no conflicts of interest to declare.

Ethical Statement: The authors are accountable for all aspects of the work in ensuring that questions related to the accuracy or integrity of any part of the work are appropriately investigated and resolved. The study was approved by the ethics committee of the Second Affiliated Hospital of Guangzhou Medical University and written informed consent was obtained from all patients.

\section{References}

1. Wood KE, Coursin DB. Intensivists and organ donor management. Curr Opin Anaesthesiol 2007;20:97-9.

2. Black CK, Termanini KM, Aguirre O, et al. Solid organ transplantation in the 21st century. Ann Transl Med 2018;6:409.

3. Chinese Society of Organ Transplantation. Organ Donation After Cardiac Death (2nd Edition). Chinese Journal of Transplantation (Electronic Version) 2012;6:221.

4. Ngaage LM, Elegbede A, Tadisina KK, et al. Panniculectomy at the time of living donor renal transplantation: An 8-year experience. Am J Transplant 2019;19:2284-93.

5. Lesieur O, Genteuil L, Leloup M. A few realistic questions raised by organ retrieval in the intensive care unit. Ann Transl Med 2017;5:S44.

6. Sawinski D, Forde KA, Lo Re V 3rd, et al. Mortality and Kidney Transplantation Outcomes Among Hepatitis C Virus-Seropositive Maintenance Dialysis Patients: 
A Retrospective Cohort Study. Am J Kidney Dis 2019;73:815-26.

7. Schnuelle P, Schmitt WH, Weiss C, et al. Effects of Dopamine Donor Pretreatment on Graft Survival after Kidney Transplantation: A Randomized Trial. Clin J Am Soc Nephrol 2017;12:493-501.

8. Benck U, Jung M, Krüger B, et al. Donor Dopamine Does Not Affect Liver Graft Survival: Evidence of Safety From a Randomized Controlled Trial. Liver Transpl 2018;24:1336-45.

9. Sun Q, Huang Z, Zhou H, et al. New Factors Predicting Delayed Graft Function: a Multi-Center Cohort Study of Kidney Donation After Brain Death Followed by Circulatory Death. Kidney Blood Press Res 2018;43:893-903.

10. Schnuelle P, Gottmann U, Hoeger S, et al. Effects of donor pretreatment with dopamine on graft function after kidney transplantation: a randomized controlled trial.

Cite this article as: Zhang $\mathrm{P}$, Cao P, Fang J, Li G, Zhang L, Xu L, Mo S, Lai X, Liu L, Xiong Y, Yin W, Li L, Chen R, Xu H, Zhang T, Wan J, Guo Y, Ma J, Chen Z. Impact of the use of vasoactive drugs in cardiac death donors on the early postoperative renal function and related complications in renal transplant recipients. Ann Transl Med 2020;8(4):116. doi: 10.21037/atm.2019.12.99
JAMA 2009;302:1067-75.

11. Schnuelle P, Schmitt WH, Weiss C, et al. Effects of dopamine donor pretreatment on graft survival after kidney transplantation: a randomized trial. Clin J Am Soc Nephrol 2017;12:493-501.

12. Birtan D, Arslantas MK, Altun GT, et al. Effect of Vasoactive Therapy Used for Brain-Dead Donors on Graft Survival After Kidney Transplantation. Transplant Proc 2018;50:1289-91.

13. O'Brien EA, Bour SA, Marshall RL, et al. Effect of use of vasopressors in organ donors on immediate function of renal allografts. J Transpl Coord 1996;6:215-6.

14. Shao M, Ye Q, Ming Y, et al. Delayed graft function after DCD kidney transplantation: risk factors for and impact on transplantation. Zhong Nan Da Xue Xue Bao Yi Xue Ban 2012;37:1045-9. 